\title{
A Nanoscale Model System for the Human Myelin Sheath
}

\author{
Matthias Hoffmann, ${ }^{1,2}$ David Haselberger, ${ }^{1,2}$ Tommy Hofmann, ${ }^{1,3}$ Lisa Müller, ${ }^{4}$ Kevin \\ Janson, ${ }^{1,3}$ Annette Meister, ${ }^{1,3}$ Manabendra Das, ${ }^{5}$ Carolyn Vargas, ${ }^{5-8}$ Sandro Keller, ${ }^{5-8}$ \\ Panagiotis L. Kastritis, ${ }^{1,3}$ Carla Schmidt, ${ }^{1,3}$ Dariush Hinderberger ${ }^{1,2, *}$
}

1 Interdisciplinary Research Center HALOmem, MLU Halle-Wittenberg, Charles Tanford Protein Center, Kurt-Mothes-Str. 3a, 06120 Halle (Saale), Germany.

2 Institute of Chemistry, Martin Luther University (MLU) Halle-Wittenberg, Von-Danckelmann-Platz 4, 06120 Halle (Saale), Germany.

3 Institute of Biochemistry and Biotechnology, Charles Tanford Protein Center, Martin Luther University (MLU) Halle-Wittenberg, Kurt-Mothes-Str. 3a, 06120 Halle (Saale), Germany.

4 Institute of Pharmacy, MLU Halle-Wittenberg, Wolfgang-Langenbeck-Str. 4, 06120 Halle (Saale), Germany.

5 Molecular Biophysics, Technische Universität Kaiserslautern (TUK), Erwin-Schrödinger-Str. 13, 67663 Kaiserslautern, Germany.

6 Biophysics, Institute of Molecular Biosciences (IMB), NAWI Graz, University of Graz, Humboldtstr. 50/III, 8010 Graz, Austria.

7 Field of Excellence BioHealth, University of Graz, $8010 \mathrm{Graz}$, Austria.

8 BioTechMed-Graz, 8010 Graz, Austria.

KEYWORDS Lipid nanodiscs, myelin, EPR spectroscopy, lipid quantification

Neurodegenerative disorders are among the most common diseases in modern society. However, the molecular bases of diseases such as multiple sclerosis or Charcot-Marie-Tooth disease remain far from being fully understood. Research in this field is limited by the complex nature of native myelin and by difficulties in obtaining good in vitro model systems of myelin. Here, we introduce an easy-to-use model system of the myelin sheath that can be used to study myelin proteins in a native-like yet well-controlled environment. To this end, we present myelin-mimicking nanodiscs prepared through one of the amphiphilic copolymers styrene/maleic acid (SMA), diisobutylene/maleic acid (DIBMA), and styrene/maleimide sulfobetaine (SMA-SB). These nanodiscs were tested for their lipid composition using chromatographic (HPLC) and mass spectrometric (MS) methods and, utilizing spin probes within the nanodisc, their comparability with liposomes was studied. In addition, their binding behavior with bovine myelin basic protein (MBP) was scrutinized to ensure that the nanodiscs represent a suitable model system of myelin. Our results suggest that both SMA and SMA-SB are able to solubilize the myelin-like (cytoplasmic) liposomes without preferences for specific lipid headgroups or fatty acyl chains. In nanodiscs of both SMA and SMA-SB (called SMA(-SB)-lipid particles, short SMALPS or SMA-SBLPS, respectively) the polymers restrict the lipids' motion in the hydrophobic center of the bilayer. The head groups of the lipids, however, are sterically less hindered in nanodiscs when compared with liposomes. Myelin-like SMALPs are able to bind bovine MBP which can stack the lipid bilayers like in native myelin, showing the usability of these simple, well-controlled systems in further studies of protein-lipid interactions of native myelin.

\section{Introduction}

In our society, medicine is frequently confronted with neurodegenerative diseases such as multiple sclerosis or the CharcotMarie-Tooth disease which are caused in part by myelination deficiency. ${ }^{1-3}$ For early- state research of diseases connected to demyelination a variety of potential model systems is currently used in molecular-level studies $^{4-8}$. All of them have their advantages and disadvantages and lead to a significant reduction of complexity compared to native myelin. This has the drawback that in 
studies of interactions between myelin lipids and myelin proteins in these model systems it is hard to know if and how the findings can be transferred to the behavior in native myelin. However, such an understanding is essential and fundamental for a deeper understanding of pathological conditions in patients.

At the molecular level, the myelin sheath wraps around the axons of nerve cells of vertebrates. $^{2}$ Its main function is the insulation of the axon from electrical activity to increase the rate of signal transmission. ${ }^{2}$ Thus, the membrane action potential is transmitted with high efficiency by saltatory conduction. ${ }^{9}$ To achieve this insulating function, the myelin sheath mainly consists of lipid bilayers (lipid content 70-80 $\%{ }^{10}$ ) that are tightly compacted by several proteins. ${ }^{11-13}$ Mutations, posttranslational modifications, and deficiencies in these proteins can lead to demyelination and neurodegenerative diseases. ${ }^{3,11}$

The rather new class of polymerencapsulated nanodiscs, ${ }^{14-15}$ which are stackable and flat, fulfills the requirements for representing a myelin sheath model system, e.g. to contain all major lipids and proteins abundant in human myelin, including high amounts of cholesterol, and others listed in the SI. In addition, the polymeric nature of the used amphiphiles prevents penetration of the bilayer which could affect lipid properties or denature incorporated proteins. The main downside to most of the known polymers that are able to solubilize lipid membranes is their strong absorption in the UV range which is useful for protein studies. ${ }^{16}$ To overcome this and other challenges, new polymers are developed constantly. ${ }^{16-18}$

In this work, we show how excellent nanoscale models for myelin lipid bilayer membranes with the composition of the CNS or PNS cytosolic leaflet can be achieved (see SI for details on criteria) by solubilization of myelin-like liposomes of the cytosolic CNS and PNS lipid composition with the well-known polymers styrene/maleic acid (SMA 2:1) $)^{19}$ and diisobutylene/maleic acid (DIBMA) ${ }^{16}$ as well as the new polymer styrene/maleimide sulfobetaine (SMA-SB). ${ }^{20-21}$ The solubilization of these lipid mixtures is remarkably challenging because of the high amount of negatively charged, unsaturated phospholipids and the exceptionally high cholesterol content. ${ }^{22-24}$ After preparation, the nanodiscs were investigated regarding their lipid composition using HPLC and MS, their lipid properties with continuous wave electron paramagnetic resonance (CW EPR), and their interactions with the model protein bMBP, which is known to act as "molecular glue" between two myelin layers in both PNS and CNS. ${ }^{11}$ The achievement of lipid nanodiscs with the challenging lipid compositions of PNS and CNS myelin can in general be viewed as paving the way for more complex lipid model membrane systems on the one hand, and in this specific case allows for future studies on protein-lipid interactions in myelin and factors enabling or even enforcing myelin formation or degradation, even using native-like combinations of myelin proteins in a realistic yet highly controlled lipid environment.

\section{Materials and Methods}

\section{Materials}

The three polymers poly-(styrene-maleic acid) (SMA 2:1 hydrolyzed from styrene/maleic anhydride (Xiran SZ30010)), poly-(diisobutylene-alt-maleic acid) (DIBMA) and poly-(styrene-maleimide sulfobetaine) (SMA-SB) were synthesized following a protocol published previously. ${ }^{20}$ HPLC-grade chloroform $\left(\mathrm{CHCl}_{3}\right)$ and methanol $(\mathrm{MeOH})$ as well as the buffer salts were purchased from Carl Roth (Karlsruhe, Germany, tris buffer salt $>99.3 \%$ and $\mathrm{NaCl}>99.7 \%$ ). Aqueous ammonia was bought from Grüssing (Filsum, Germany). All used lipids were purchased from Avanti Polar Lipids (Alabaster, AL, USA, for further details see Figure $\mathbf{S 1}$ in the Supporting Information). The model protein bMBP $(\geq 90 \%)$ was purchased from Sigma (St. Louis, MO, USA).

Preparation of polymer solutions and polymer-lipid samples

Polymer stock solutions were prepared as previously described. ${ }^{20-21}$ An appropriate amount of either SMA or SMA-SB was dissolved in saline tris buffer $(50 \mathrm{mM}$ tris, $300 \mathrm{mM} \mathrm{NaCl}, \mathrm{pH} 7.4$ in Millipore MilliQ water with a specific resistance of $\rho=18.2 \mathrm{M} \Omega \mathrm{cm}$ ) to yield mass concentrations of $25 \mathrm{mg} / \mathrm{mL}$ (SMA) or $15 \mathrm{mg} / \mathrm{mL}$ (SMA-SB) at room temperature followed by heating combined with ultrasonicating for $30 \mathrm{~min}$ at $70^{\circ} \mathrm{C}$. After cooling down, both polymer stock solutions were stable for two weeks at room temperature. DIBMA stock solutions were prepared as described before. ${ }^{16}$ In short, Sokalan CP9 (BASF, Ludwigshafen, Germany) was dialyzed against tris buffer and the DIBMA concentration was checked via refractive index with an Abbemat 450 (Anton Paar, Graz, Austria) using a literature ${ }^{16}$ value for $\mathrm{d} n / \mathrm{d} c$.

Preparation of myelin-like nanodiscs was conducted as follows: the lipids containing 
either no or $3.3 \mathrm{~mol} \%$ spin label lipid (5DSPC or 16DSPC, replacing PC lipids in part) were dissolved in $\mathrm{CHCl}_{3} / \mathrm{MeOH} 2: 1$ (vol/ vol), mixed in lipid compositions resembling cytosolic CNS (cholesterol:PE:PS:PC:SM:PI 44:27:13:11:3:2) or PNS (cholesterol:PE:PS:PC:SM:PI 37:22:19:9:9:4) myelin. ${ }^{25-26}$ Subsequently, the solvent was evaporated in a gentle stream of nitrogen to obtain a thin dry lipid layer. Tris buffer was added, and the total lipid concentration was adjusted to somewhat above $3 \mathrm{mM}$. The lipids were allowed to suspend at $45^{\circ} \mathrm{C}$ while ultra-sonicating the sample for at least $10 \mathrm{~min}$. Subsequently, the resulting liposomes were extruded (35 times) through a $400 \mathrm{~nm}$ polycarbonate membrane. We have chosen to use liposomes larger than $100 \mathrm{~nm}$ to increase the size difference between nanodiscs and remaining liposomes and, thus, to improve the chromatographic separation in the next step. Prepared liposomes were mixed with polymer solutions to various polymer to lipid ratios at a final lipid concentration of $3 \mathrm{mM}$ and, subsequently, incubated at $45^{\circ} \mathrm{C}$ for $16 \mathrm{~h}$ while shaking.

Separation of nanodiscs and remaining liposomes

During solubilization of liposomes of a myelin-like lipid composition, the resulting nanodiscs had to be separated from remaining liposomes which could hinder downstream characterization techniques. For this, $500 \mu \mathrm{L}$ of the $3 \mathrm{mM}$ lipid/polymer suspensions were injected onto a Superose 6 Increase 10/300 GL size exclusion chromatography (SEC) column (GE Healthcare Bio-Sciences AB, Uppsala, Sweden) mounted to an Äkta pure (GE Healthcare Bio-Sciences AB) chromatography system. The following size exclusion chromatography was conducted using saline tris buffer ( $50 \mathrm{mM}$ tris, $300 \mathrm{mM}$ $\mathrm{NaCl}, \mathrm{pH}$ 7.4) for elution with a flow rate of $0.5 \mathrm{~mL} / \mathrm{min}$. After discarding approximately $7.5 \mathrm{~mL}(5 \mathrm{~mL}$ injection loop purging and discarding of the first $2.5 \mathrm{~mL}$ which is 0.1 column volumes (CVs)) fractions of $500 \mu \mathrm{L}$ each were collected until 1.1 CVs were eluted. Detection during chromatography was conducted with UV absorbance detectors operating at $254 \mathrm{~nm}$ for samples containing SMA or SMA-SB and at $220 \mathrm{~nm}$ for DIBMA. The fractions within UV absorbance peaks were further studied with dynamic light scattering to ensure the presence of particles in the size range typical for lipid nanodiscs.

Dynamic light scattering (DLS)

DLS experiments were conducted on a Litesizer 500 (Anton Paar) either with a
$70 \mu \mathrm{L} 3 \mathrm{~mm} \times 3 \mathrm{~mm}$ Micro Quartz cuvette (Hellma, Müllheim, Germany) or disposable cuvettes of $500 \mu \mathrm{L}$, the latter for characterization of SEC fractions. The irradiation wavelength was $\lambda=658 \mathrm{~nm}$ while the detection angle was maintained at $90^{\circ}$ (side scattering) or $175^{\circ}$ (back scattering) at constant temperature of $20{ }^{\circ} \mathrm{C}$ if not stated otherwise. Prior to each measurement, the sample was allowed to equilibrate for at least $1 \mathrm{~min}$. Data obtained from DLS measurements was evaluated directly using the Kalliope software (Anton Paar).

Transmission electron microscopy (TEM)

TEM samples were prepared by spreading $5 \mu \mathrm{L}$ of the (polymer-)lipid suspensions after chromatographic separation of remaining liposomes and dilution with an equal amount of tris buffer onto freshly glow discharged grids with carbon support film (300 mesh) on copper (Quantifoil Micro Tools, Großlöbichau, Germany). After 30$45 \mathrm{~s}$ excess suspension was blotted up using filter paper. The grids were washed three times with MilliQ water. $5 \mu \mathrm{L}$ of $2 \%$ aqueous uranyl acetate solution was placed onto the grid and also blotted up after $1 \mathrm{~min}$. After preparation the samples were dried for at least $24 \mathrm{~h}$. All TEM samples were examined with a Zeiss EM 900 transmission electron microscope (Carl Zeiss Microscopy, Jena, Germany). In addition, the best sample was also imaged using a Glacios $200 \mathrm{kV}$ cryo-electron transmission microscope (Thermo Fisher Scientific, Eindhoven, Netherlands). The respective images were acquired on a Falcon 3EC direct electron detector in linear mode with a total dose of $30 \mathrm{e}^{-} / \AA^{2}$, and a magnification of $92000 \mathrm{X}$, resulting in a pixel size of $1.567 \AA /$ pixel.

\section{Extraction of lipids with organic solvent}

For lipid quantification and mass spectrometric analysis, lipids of the prepared suspensions were extracted to $\mathrm{CHCl}_{3} / \mathrm{MeOH}$ following the protocol of Bligh and Dyer. ${ }^{27}$ In short, to the volume of the aqueous suspension $\mathrm{MeOH}$ ( $2.5 \mathrm{x}$ volume) and $\mathrm{CHCl}_{3}$ (1.25x volume) were added and the mixture was shaken for $2 \mathrm{~min}$. Again, $\mathrm{CHCl}_{3}$ (1.25x volume) was added followed by shaking for $30 \mathrm{~s}$. Additional water was added ( $1.25 x$ volume) and once again the mixture was shaken for $30 \mathrm{~s}$. The layers were allowed to separate and the lower layer containing mainly $\mathrm{CHCl}_{3}, \mathrm{MeOH}$, and nonpolar lipids was collected and the solvents were evaporated in a gentle stream of nitrogen.

Lipid quantification with high performance liquid chromatography (HPLC) 
Lipids in nanodisc and liposome samples were quantitatively analyzed using an HPLC method customized from the literature. ${ }^{28}$ The measurement system contained a LC Net II/ADC Interface Box, a PU-980 pump, a LG-2080-02 gradient mixer and a DG-208053 3-line degasser (all from JASCO Deutschland, Pfungstadt, Germany) as well as a LiChrospher Si $60(5 \mu \mathrm{m})$ LiChro-CART 125-4 column (Merck, Darmstadt, Germany) and a SEDEX 55 ELS detector (SEDERE, Alfortville, France). Prior to sample measurements, stock solutions containing all lipids in appropriate ratios were prepared to calibrate the quantification (see FIG S6 and S7). $20 \mu \mathrm{L}$ sample were injected for each measurement. Elution was conducted at $1 \mathrm{~mL} / \mathrm{min}$ with a solvent gradient of (A) $80 / 19.5 / 0.5 \quad \mathrm{CHCl}_{3} / \mathrm{MeOH} / \mathrm{NH}_{3, \mathrm{aq}}(32 \%)$ and (B) $60 / 34 / 5.5 / 0.5 \quad \mathrm{CHCl}_{3} / \mathrm{MeOH} /$ $\mathrm{H}_{2} \mathrm{O} / \mathrm{NH}_{3, \mathrm{aq}}(32 \%$ ) (all solvent mixtures in vol $\%)$ following literature. ${ }^{28}$ During elution, the solvent gradient was adjusted linearly as follows (here shown as relative content of solvent A): $0-14 \min 100 \% \rightarrow 0 \%$; 14$23 \min 0 \%$; $23-24 \min 0 \% \rightarrow 100 \%$; and 24-25 min $100 \%$.

Mass spectrometry (MS)

Lipids were extracted from the nanodiscs and spotted onto a $10 \times 10 \mathrm{~cm}$ silica gel 60 thin layer chromatography (TLC) plate F254 (Merck). TLC separation was performed in $\mathrm{CHCl}_{3} / \mathrm{MeOH} / \mathrm{H}_{2} \mathrm{O}$ (65:25:4) as mobile phase. After separation, TLC plates were air-dried and stained with a $0.03 \%(w / v)$ Coomassie Brilliant blue G250 solution in $20 \%(\mathrm{v} / \mathrm{v})$ $\mathrm{MeOH}$ and $80 \% \mathrm{H}_{2} \mathrm{O}$. Visible spots were removed from the carrier and stained lipids extracted as previously described. ${ }^{29}$

For lipid quantification, dried lipids were dissolved in $20 \mu \mathrm{l}$ of $80 \% \mathrm{MeOH} / 20 \% \mathrm{CHCl}_{3}$ $(v / v)$ and $2 \mu \mathrm{l}$ of a 100 times diluted stock of SPLASH Lipidomix Mass Spec standard (Sigma-Aldrich) in $80 \% \mathrm{MeOH} / 20 \% \mathrm{CHCl}_{3}$ was added to each sample.

Mass spectra were acquired on a Q Exactive Plus Hybrid Quadrupole-Orbitrap Mass Spectrometer (Thermo Fisher Scientific, Waltham, MA, USA) equipped with a Nanospray Flex ion source (Thermo Fisher Scientific). $5 \mu \mathrm{l}$ of sample were loaded into borosilicate offline emitter coated with gold/ palladium (Thermo Fisher Scientific).

MS settings were as follows: capillary voltage, \pm 2.5 to $2.8 \mathrm{kV}$; capillary temperature, $250{ }^{\circ} \mathrm{C}$; resolution, 70000 ; RFlens level, 50; scan range of MS spectra, 400-1600 $\mathrm{m} / \mathrm{z}$; ion mode, positive (for PC lipids) or negative (for PE/PS/PI lipids); maximal injection time, $100 \mathrm{~ms}$; automatic gain control target, $2 \cdot 10^{6}$; microscans, 1 ; target resolution, 100000 .
Tandem MS settings: maximal injection time, $100 \mathrm{~ms}$; automatic gain control target, $5 \cdot 10^{5}$; microscans, 1 ; target resolution, 70000; fragmentation type, HCD; mass selection window, $1 \mathrm{~m} / \mathrm{z}$; Top20 automatic precursor selection for $1 \mathrm{~min}$; selected precursors were excluded for $30 \mathrm{~s}$.

Acquired mass spectra were analyzed manually using the lipid maps tools (http://www.lipidmaps.org/tools/).

Continuous wave electron paramagnetic resonance (CW EPR) spectroscopy

CW EPR spectroscopy was used to further characterize the impact of surrounding polymers on the lipids while being able to differentiate between different polarities or restricted rotational motions. To this end, spin-labeled lipids (5DSPC or 16DSPC, $3.3 \mathrm{~mol} \%$ of total lipid) were incorporated in the liposomes (and, therefore, the nanodiscs solubilized from the liposomes) substituting only phosphocholine lipids. To ensure a good signal to noise ratio the aqueous suspensions after SEC were concentrated using centrifugation filters (Amicon Ultra 4 with a molecular weight cutoff of $30 \mathrm{kDa}$, Merck Millipore, Billerica, MA, USA) at $3220 \times g$ until a volume of 200 $300 \mu \mathrm{L}$ was reached. After centrifugation, the presence of nanodiscs in the resulting suspensions were checked using DLS once again. $10-15 \mu \mathrm{l}$ of the sample were filled into micropipettes (BLAUBRAND intraMark, Wertheim, Germany) and capped with capillary tube sealant (CRITOSEAL Leica, Wetzlar, Germany).

$X$-band CW EPR spectroscopic measurements were performed with the Miniscope MS5000 (magnettech, Freiberg Instruments, Freiberg, Germany) benchtop spectrometer. Temperature control was achieved by connecting the $\mathrm{HO} 4$ temperature control unit (magnettech) and, thus, temperature series were recorded between $0-70{ }^{\circ} \mathrm{C}$. All spectra were measured with a modulation amplitude of $0.1 \mathrm{mT}$ and a microwave power of $3.63 \mathrm{~mW}$. The range of the magnetic field was adjusted to the width of measured spectra and set between 332.5 and $347.5 \mathrm{mT}$.

Analysis of the recorded CW EPR spectra was conducted using MatLab (R2017a, v. 9.2) in combination with the EasySpin toolbox (v. 5.2.28) for EPR spectroscopy. ${ }^{30}$ Spectra of liposomes (for both 16DSPC and 5DSPC) and all other systems containing 5DSPC were simulated with a single component using the model of anisotropic Brownian motion with an axial symmetry and orienting potential (see Supporting Information for further information). ${ }^{31-32}$ In nanodisc systems containing 16DSPC, 
inclusion of a second component was necessary to achieve an acceptable agreement between simulated and experimental spectra.

Differential scanning calorimetry (DSC)

To analyze the impact of the three polymers on macroscopic lipid phase transitions, DSC was used without chromatographic separation of liposomes, since both lipid mixtures did not show any phase transitions in liposomes. Prior to all measurements, the samples as well as the reference buffer solution was degassed under vacuum while stirring. DSC measurements were performed using a MicroCal VP-DSC differential scanning calorimeter (MicroCal, Northampton, MA, USA). In all experiments, 5 heating/cooling cycles were measured to assure sample stability and reproducibility from which one representative heating curve (heating rate $60 \mathrm{~K} \mathrm{~h}^{-1}$ between 5 and $95{ }^{\circ} \mathrm{C}$ ) was chosen. Further evaluation of the DSC results involved subtraction of regularly collected buffer/buffer baselines as well as subtraction of offset values, if necessary.

\section{Results and Discussion}

\section{Preparation of myelin-like nanodiscs}

The successful preparation of myelin-like nanodiscs requires knowledge of the optimal polymer/lipid ratio for optimal solubilization. ${ }^{19}$ To achieve this, the solubilization efficiency of the three polymers towards myelin-like liposomes was tested with DLS by measuring autocorrelation functions as exemplarily shown in Figure 1A. For all other autocorrelation functions see Figure S2. Solubilization of the liposomes goes along with a decrease in the size of the scattering particles and, thus, with a shift of the autocorrelation function to shorter correlation times. ${ }^{16,}{ }^{19}$ In previous studies using polymer nanodiscs, the solubilization efficiency was tested determining either the size of particles, ${ }^{16,19}$ the turbidity of the sample, $^{15}$ or other methods. ${ }^{16-17,} 19$ Here, both of the former methods were found unsuitable for detecting the presence of nanodiscs because only partial solubilization of the complex lipid mixtures could be achieved. The decreased solubilization efficiency is easily explained by the lipid mixtures used here. ${ }^{22-24}$ It is known that solubilization works best with saturated short-chain lipids such as DMPC and if unsaturated lipids are used, solubilization efficiency is reduced. ${ }^{19,} 23$ This is thought to be due to an increased lateral pressure within the lipid bilayer, which impedes the penetration of the amphiphilic polymers into liposomes. ${ }^{22-23}$ In addition, lipid mixtures studied here contain large amounts of cholesterol, which is also known to increase the lateral pressure of the bilayer ${ }^{33}$ and, furthermore, causes the bilayer to phaseseparate into ordered and disordered domains. ${ }^{34}$ This phase separation may hinder the complete solubilization process as well, given that domain formation is possible in liposomes but probably not in the size-restricted nanodiscs because of the much smaller number of lipids they contain.

All optimal polymer/lipid ratios $(w / w)$ for the solubilization approach used here are shown in Table S1. As can be concluded from DLS measurements (see Figure S2), SMA and SMA-SB showed substantial shifts in the autocorrelation function, whereas DIBMA exhibited only minor effects. While for solubilization of CNS liposomes only small polymer/lipid ratios were necessary in the case of SMA (1.5/1) and SMA-SB (2/1), solubilization of PNS liposomes was most effective when all three polymers were added in the highest amount suitable for the following SEC, i.e. at a ratio of polymer/lipid of $5 / 1$.

Since the liposomes cannot be solubilized completely, the nanodiscs have to be separated from the remaining liposomes. In this study, the separation was conducted by SEC.

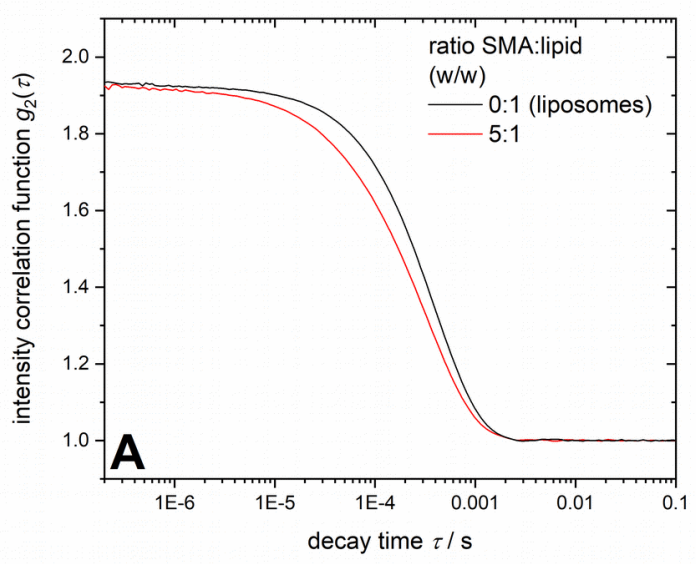



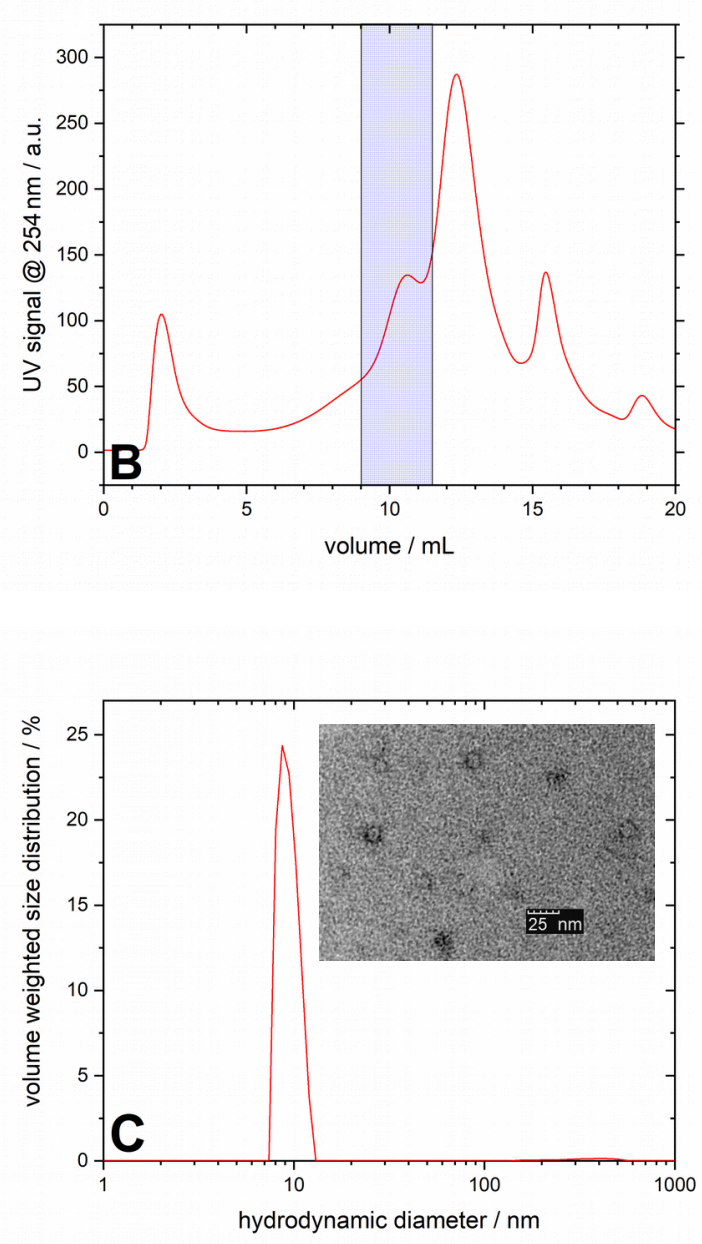

Figure 1. Preparation of myelin-like SMALPS containing lipids of the cytosolic PNS myelin composition. (A) Intensity correlation functions before and after addition of SMA to PNS liposomes (here, a lipid concentration of $1 \mathrm{mM}$ was used); (B) size exclusion chromatogram of the resulting mixture of liposomes, SMA, and SMALPs with marked fractions containing nanodiscs conducted with a flow rate of $0.5 \mathrm{~mL} / \mathrm{min}$; (C) size characterization of PNS SMALPs with DLS side scattering at $20^{\circ} \mathrm{C}$, the inset in (C) shows a section of a corresponding TEM micrograph with nanodiscs marked by yellow arrows.

The detailed chromatographic separation and purification of all nanodisc-forming systems can be found in the SI. Comparison of all three polymers leads to the conclusion that only in lipid/SMA systems an additional peak appears in the chromatograms originating from nanodiscs. For both SMA-SB and DIBMA, possible nanodisc peaks are overlaid by a peak of pure polymer.

After SEC, all collected fractions that showed UV absorption were further tested for their particle size using DLS. If particles in the range of lipid nanodiscs $(5-30 \mathrm{~nm})$ were found, the respective fractions were combined (compare Figure $\mathbf{1 C}$ and Figure S4). The nanodisc sizes observed in DLS correlate well with the elution volume of the samples in the SEC when comparing all polymers. Note that in all fractions remaining liposomes were detected even after SEC. This may be the result of a low separation efficiency of the SEC but, since the chromatograms did not show a significantly increased baseline, an equilibrium between nanodiscs and liposomes, even after separation, is more probable. From literature it is known that polymer-encapsulated nanodiscs show fast lipid exchange among each other and in combination with monolayers. ${ }^{35-37}$ A similar but somewhat slower lipid exchange would probably be observed between nanodiscs liposomes, too. Thermodynamically, the driving force for solubilization of liposomes to nanodiscs is described using transfer free energies of both the polymer or the lipid molecules. ${ }^{38-39} \Delta G_{\text {lipid }}$ is positive, that is the lipids prefer their initial bilayer state within the liposome, while $\Delta G_{\text {polymer }}$ is negative, i.e., the polymers prefer surrounding the nanodisc rather than being adsorbed to liposomes. ${ }^{19,}$ 38-39 In case of the complex lipid mixtures, used in this study, the magnitudes of both transfer free energies could be in a range which allows an equilibrium between nanodiscs and liposomes containing polymer to be detectable. This is plausible especially for mixtures containing a high amount of cholesterol because in liposomes these lipid mixtures are known to form ordered domains. In nanodiscs, by contrast, their size restrictions probably inhibit the formation of sizeable domains and, thus, $\Delta G_{\text {lipid }}$ could be significantly more positive. For future applications of myelin-like nanodiscs, a further thermodynamic evaluation will be necessary, which could be conducted using e.g. ${ }^{31} \mathrm{P} \quad \mathrm{NMR}$ spectroscopy. ${ }^{38-39}$

From these lipid/polymer suspensions, TEM samples were prepared by diluting them to $50 \%$ of their initial concentration and ensuing preparation steps (see Materials and Methods). TEM micrographs were collected to confirm the presence of nanodiscs after separation of remaining liposomes. 

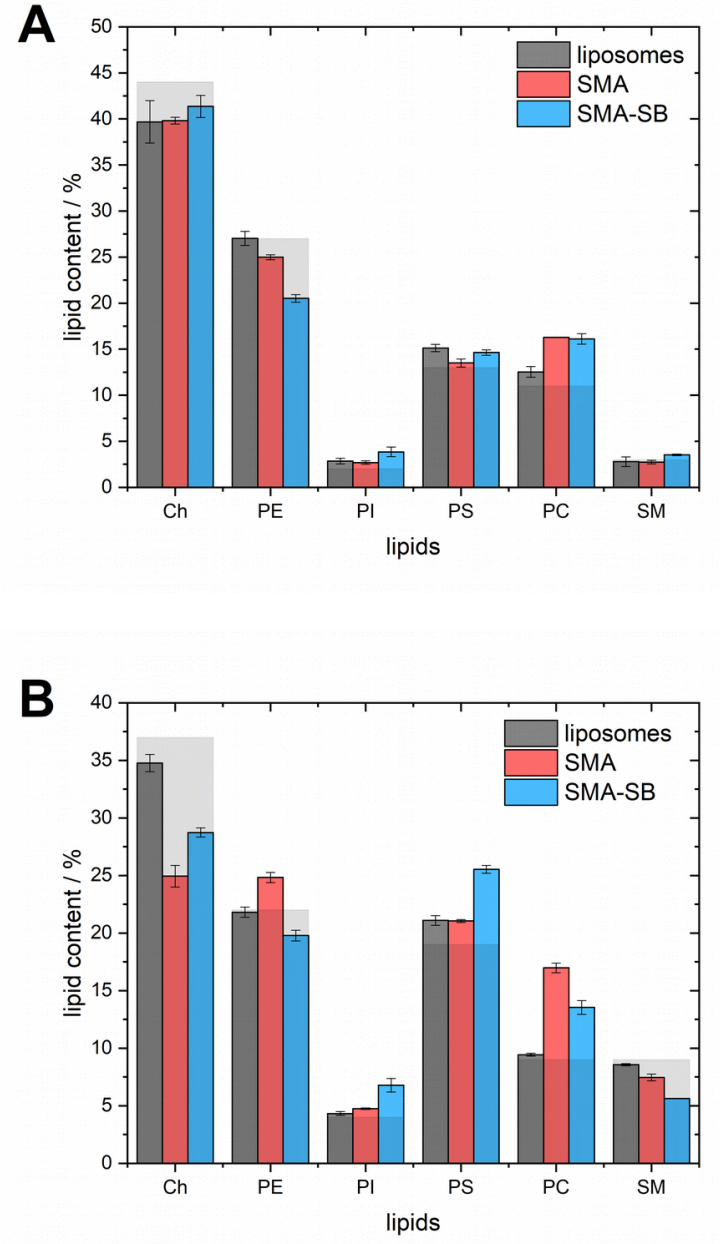

Figure 2. Lipid composition of myelin-like nanodiscs as detected with HPLC after extraction with $\mathrm{CHCl}_{3} / \mathrm{MeOH}$; (A), cytosolic CNS lipid mixture, (B), cytosolic PNS lipid mixture. Light gray columns represent the theoretical lipid composition used for liposome preparation. The lipids are shown in order of their appearance in the chromatograms and error bars represent the standard error of three independent experiments.

The TEM micrographs (see Figure S5 in the Supporting Information) of all samples show the successful preparation of myelin-like nanodiscs. However, the three studied polymers differ in contrast. Nanodiscs of SMA and DIBMA yield clear micrographs with sufficient contrast, while nanodiscs containing SMA-SB appear to have lower contrast. This is probably due to the different ionic character of the polymers, as SMA and DIBMA are anionic, whereas SMA$\mathrm{SB}$ is zwitterionic, ${ }^{20}$ which may influence the binding affinity of the contrast agent uranyl acetate. To ensure constant staining for all polymers, we optimized the staining procedure and prepared the samples three time with each preparation being a double determination.
The size of the detected nanodiscs correlated well with the hydrodynamic diameter measured with DLS for all samples.

The whole preparation process is exemplarily shown for the system PNS lipids with SMA (polymer/lipid 5/1) in Figure 1.

Analysis of lipid properties in myelin-like nanodiscs

After successful preparation of nanodiscs of myelin-like liposomes, their lipid contents were studied to ensure similarity with the well-known model system of liposomes. This is because, although the lipid vesicles were formed to resemble the myelin sheath composition, it is unknown if, during solubilization, nanodisc lipid composition was robustly recovered.

To elucidate compositional variation, the lipid composition of prepared myelin-like nanodiscs was studied with analytical HPLC regarding headgroup content (see Figure S8). ${ }^{28}$ In Figure 2 the composition of each system is shown in comparison to the lipid composition of liposomes prior to solubilization with the polymers. Only SMALPs and SMA-SBLPs are shown, as DIBMALPs yielded no reproduceable results, which may be due to the low solubilization efficiency of DIBMA (see chapter following the lipid analysis).

From Figure 2 it can be concluded that the additional extraction steps ${ }^{27}$ induce only minor changes in detected lipid compositions (compare the theoretical composition shown in light gray and liposomes). Both SMA and SMA-SB solubilize all lipids in similar amount as they are present in the liposomes and only minor preferences are observed which is in accordance with literature. ${ }^{22,} 40$ Somewhat stronger deviations from liposomal compositions were found in the case of the PNS lipid mixture: while SMA appears to show a minor deficiency in cholesterol content and a higher amount of PC, SMA-SB prefers to solubilize anionic lipids. The latter effect was also observed in the CNS lipid mixture but to a smaller extent. For other lipid mixtures containing even more negatively charged lipids, this solubilization preference of SMA-SB could be useful since the mainly used negatively charged polymers SMA and DIBMA showed repulsive interactions with anionic molecules. ${ }^{20,}$ 41-42 However, deviations from the liposomal lipid compositions were only minor and, hence, myelin-like nanodiscs can be assumed to exhibit similar surface properties as myelinlike liposomes in general.

Besides the lipid composition in terms of surface charge, i.e., the relative ratio of all 
headgroups, the polymers could prefer different lipid chain compositions as well. To study lipid chain composition, we used mass spectrometry after extraction and TLC. ${ }^{29}$ TLC was conducted to minimize overlap of lipid species with similar mass in the acquired mass spectra. ${ }^{29}$ The results of MS analysis of all systems are shown in Figure $\mathbf{S 9 .}$

For $\mathrm{PE}, \mathrm{PS}$, and $\mathrm{PI}$, all myelin-like nanodiscs exhibited similar chain compositions as observed in liposomes of the same lipid mixture. For PC lipids, in contrast, deviations are found depending on the polymer and the lipid mixture. These stronger deviations can be attributed to the poor signal/noise ratio of PC lipids in this experiment resulting from polymeric contamination that could not be removed. For all but few exceptions all lipids were found in both liposomes and nanodiscs of all three polymers. Therefore, it can be concluded, that SMA, SMA-SB, and DIBMA do not exhibit pronounced preferences for distinct types of lipid chains.

Besides lipid preferences, the polymers were also compared concerning their lipid solubilization efficiency. As can be concluded from Figure S2, SMA and SMASB exhibited strong effects on the diffusional correlation time, that is correlated to the mean size of the particles, as observed with DLS, whereas DIBMA did not change the mean diffusion coefficient of the scattering particles. As an additional indicator of solubilization efficiency, 16DSPC was added to the lipid mixture as spin probe. By measuring CW EPR spectra of all nanodisc systems after equal sample treatment below power saturation the spin probe concentration and, thus, the lipid concentration can be compared by measuring the double integral (DI) of the spectra. ${ }^{43-44}$ This, obviously, is possible only because none of the polymers exhibits strong preferences for either distinct lipid headgroups or distinct lipid chains. In addition, the comparability of all samples was ensured by concentrating them to similar volumes prior to measurement as well as by working with low microwave power to prevent power saturation. ${ }^{43-44}$ However, it was not possible to prepare all samples completely equal and small preferences in lipid solubilization were observed before. Therefore, the normed double integral values shown in Figure S10 have to be considered to be an approximation of lipid content. From measured double integral values, it can be concluded that only SMA and SMA-SB are capable of solubilizing PNS and CNS lipid mixtures to a suitable extent. DIBMA, in contrast, did not solubilize either of the two lipid mixtures within the experimental procedures applied in this study to an extent usable for most applications. Thus, DIBMA will be excluded from the following discussion. Nevertheless, temperaturedependent CW EPR spectroscopic measurements were also performed with DIBMALPs and are shown in the Supporting Information (see Figures S14 and S22).

CW EPR spectroscopy also enables us to unravel changes in lipid environment induced by the polymers at different positions within the bilayer. ${ }^{45-48}$ For this, spin labeled lipids were introduced bearing a doxyl moiety either in terminal position (16DSPC) or near the carbonyl group (5DSPC) of the sn-2 chain. A different position of the unpaired electron spin allows detecting hydration and rotational mobility in a distinct section of the bilayer. ${ }^{45-48}$ In this work, temperature-dependent EPR spectra were measured between 0 and $70{ }^{\circ} \mathrm{C}$ of all studied nanodisc systems as well as myelinlike liposomes and simulated with the model of Brownian motion with axial symmetry (see Supporting Information for further details and Tables S2-S13, Figure S18, and Figure $\mathbf{S 2 6}$ for simulation results). The temperature-dependent spectra and comparison of measured and simulated spectra are shown in Figures S11-S17 and Figures S19-S25.

First, from the observed CW EPR spectra, it is obvious that only minor differences between the CNS and the PNS lipid composition were found for all studied systems. The hydration, as concluded from simulated ${ }^{14} \mathrm{~N}$ hyperfine coupling constants $a_{\text {iso }}$ (see Figure S18 and Figure S26), as well as the rotational behavior (Figure 3 as well as Figure $\mathbf{S 1 8}$ and Figure S26) remained similar when comparing both CNS and PNS composition for each model system. Subsequently, in the following all results apply for both systems if not stated otherwise.

Second, due to use of 16- and 5DSPC, the nanodisc systems can be compared to liposomes of a similar composition to study the impact of each polymer onto (i) the core of the bilayer and (ii) the interface between hydrophobic chain and hydrophilic headgroup, respectively. ${ }^{45-48}$

For (i), 16DSPC was introduced into the lipid mixture prior to liposome solubilization. From the data shown in Figure $\mathbf{S 1 8}$ it can be concluded, that all studied model systems exhibit axial rotation. The rotational correlation time $\tau_{c}$ decreases with temperature. Addition of either of the two polymers SMA or SMA-SB induces an increase of $\tau_{c}$. Furthermore, the rotational 
motion of 16DSPC changes its anisotropy $T_{\text {rot }}{ }^{49}$ with increasing temperature in myelinlike liposomes while in both polymerencapsulated nanodiscs it remains nearly constant over the whole temperature range studied. Thus, both polymers hindered a more isotropic motion of the lipid chains. The CW EPR spectra were simulated using the least number of spectral components possible, that is one for liposomes and one or two for both SMALPS and SMA-SBLPS depending on temperature. Inclusion of a second component characterized by a higher hydration and lower rotational mobility, i.e., increased $a_{\text {iso }}$ as well as higher $\tau_{c}$ compared to the main component found for all systems, respectively, in both nanodisc systems was necessary to achieve a reasonable fit to the experimental data. However, the respective additional components of both polymers differ in their temperature stability, rotational restrictions, and polarity of their environment (see Figure S18). Complex spectra with correlated $a_{\text {iso }}$ and rotational behavior causes a hindered separation of the simulation parameters. The decreased $a_{\text {iso }}$ shown in Figure S18, therefore, has to be considered most probably an artifact resulting from simulation of the motional regime of the spin probe which is especially difficult to simulate in combination with environmental polarity and an additional component. However, if this is a real effect, it could in the future be studied further using hyperfine spectroscopy in $\mathrm{D}_{2} \mathrm{O}$ solvent, for example.

For lipid bilayers surrounded by SMA, an additional component between approximately $0{ }^{\circ} \mathrm{C}$ and $34{ }^{\circ} \mathrm{C}$ is needed in the analysis, which exhibits increased $\tau_{c}$ combined with a higher order parameter and a more polar spin label environment when compared with myelin-like liposomes (see Figure S18). 16DSPC in SMA-SBLPS had to be simulated with an additional component between $0{ }^{\circ} \mathrm{C}$ and $55^{\circ} \mathrm{C}$ (CNS) or $64{ }^{\circ} \mathrm{C}$ (PNS) which is denoted by even stronger rotational restrictions combined with increased order but an environmental polarity in between liposomes and SMALPs (see Figure S18). In contrast to 16DSPC in SMALPs, the spectra of 16DSPC in SMASBLPs at $0^{\circ} \mathrm{C}$ and $5^{\circ} \mathrm{C}$ can be simulated using only the additional component and, therefore, differ substantially from the spectra found in liposomes.

The additional spectral component, featuring increased polarity and hydration in each model system, can be consistently explained with the addition of the amphiphilic polymers, i.e., SMA ${ }^{14,} 42$ and $\mathrm{SMA}-\mathrm{SB}^{, 20-21}$ that change polarity directly plus disrupt the bilayer structure by providing the basis for increased water penetration into the core of the membrane. ${ }^{15-16,} 50$ On the other hand, increased restrictions of spin label rotational mobility can either be the result of a polymer-induced formation of a condensed lipid phase ${ }^{21,} 51$ or by lipid-polymer interactions with the surrounding polymer itself. ${ }^{15}, 47,50$ To study this effect, we performed DSC measurements of SMALPS and SMA-SBLPs of both lipid compositions prior to separation of the liposomes.

$C_{\mathrm{p}}$ peaks in the DSC results would indicate a lipid phase transition, and as is observable in Figure S27, in our DSC experiments there are no $C_{p}$ peaks over the whole temperature range. Furthermore, when studying 5DSPC in the same systems, no additional spectral component is necessary for suitable fit of simulation to the experimental spectra.

From the combination of CW EPR and DSC, it can be concluded that the observed spectral component with increased rotational restrictions and polarity has to be induced by direct interactions between the polymers and the lipids. Subsequently, the main difference between both polymers is probably induced by different nanodisc sizes. The prepared myelin-like SMALPs of both lipid compositions exhibited larger diameters as observed with DLS and TEM than SMA-SBLPs. Since the interfacial area between the polymer and lipids, which determines the effects visible in the additional spectral component depends on the particle diameter, it is possible that the observed interactions originate from a temperature-dependent size increase of the nanodiscs. Such an increasing size, however, was observed only for myelin-like SMALPs, while SMA-SBLPs exhibited a nearly constant size as determined by DLS (see Figure S28 and Figure S29).

Addition of 5DSPC to the lipid mixtures prior to liposome solubilization, enables the study of the interfacial region between lipid headgroups and chains. ${ }^{45-48}$ The simulation results of temperature-dependent CW EPR spectra are shown in Figure S26. They indicate only minor effects when compared with results of 16DSPC simulation which is due to the already reduced rotational mobility of the spin label. ${ }^{31,47}$ However, a change in polarity was found for SMA in comparison with liposomes at elevated temperatures. SMA shows a decreased $a_{\text {iso }}$ between $30^{\circ} \mathrm{C}$ and $65^{\circ} \mathrm{C}$ compared with liposomes, which declines again around $70{ }^{\circ} \mathrm{C}$. This could be the result of SMALPs being more hydrophobic. The differences in 
$a_{\text {iso, however, are only minor. Thus, this }}$ interpretation has to be reviewed using hyperfine spectroscopic methods in future studies. The rotational correlation time $\tau_{c}$ was affected to a small extent by the surrounding polymer, in contrast to the observations in the membrane core. Nevertheless, differences between liposomes and both nanodisc systems emerge when comparing the anisotropy of rotation $T_{\text {rot. }}{ }^{49}$ All three systems, in general, exhibited strong anisotropic motion. With increasing temperature, $T_{\text {rot }}$ of both 5DSPC in SMALPs and in SMA-SBLPs deviates from $T_{\text {rot }}$ found in liposomes to a more isotropic rotational behavior. This effect was more pronounced in the case of SMA-SBencapsulated lipid nanodiscs, which is probably due to SMA-SB being more apolar ${ }^{20}$ and, hence, preferably interacting with the hydrophobic core instead of the whole bilayer. Thus, less rotational restrictions were exerted by SMA-SB in the only weakly interacting hydrophilic part of the bilayer. As the hydrophobic-hydrophilic interfacial region remains sterically demanding and the lipids are unable to leave the bilayer, the rotation becomes more isotropic instead of becoming faster. Changes in $T_{\text {rot, }}$ as observed here, are caused by significant differences in rotational behavior while a similar $\tau_{\mathrm{c}}$ is maintained within the dataset (compare with Ref. ${ }^{47}$ ). Our results, thus, have to be considered a clear argument for changing rotational restrictions, that is an increased wiggling motion of the spin label in-plane compared to liposomes.

From studying myelin-like nanodiscs in comparison with liposomes, it can be concluded, that a variation of labeling position within the bilayer yields vastly contrary results. While both polymers induced similar effects in both the membrane core as well as the carbonyl-near region, respectively, the deviations from liposomes were more pronounced in SMASBLPs. Addition of the polymer induced increased rotational restrictions in the membrane core that were accompanied by an increase in polarity and hydration, while the rotational anisotropy in the more polar regions of the membrane was reduced. Both findings lead to a model depicted in which combines a compression effect in the hydrophobic parts with a small loosening effect in the hydrophilic parts of the membrane. SMA exhibits the same effects to a smaller extent, which is due to (i) a larger diameter of the nanodiscs resulting in a reduced interaction area between polymer and lipids in relation to lipid number and (ii) a higher polarity of the SMA polymer in comparison to SMA-SB, which enables SMA to bind both the hydrophilic and the hydrophobic areas of the lipid membrane while SMA-SB preferably binds the hydrophobic core. 

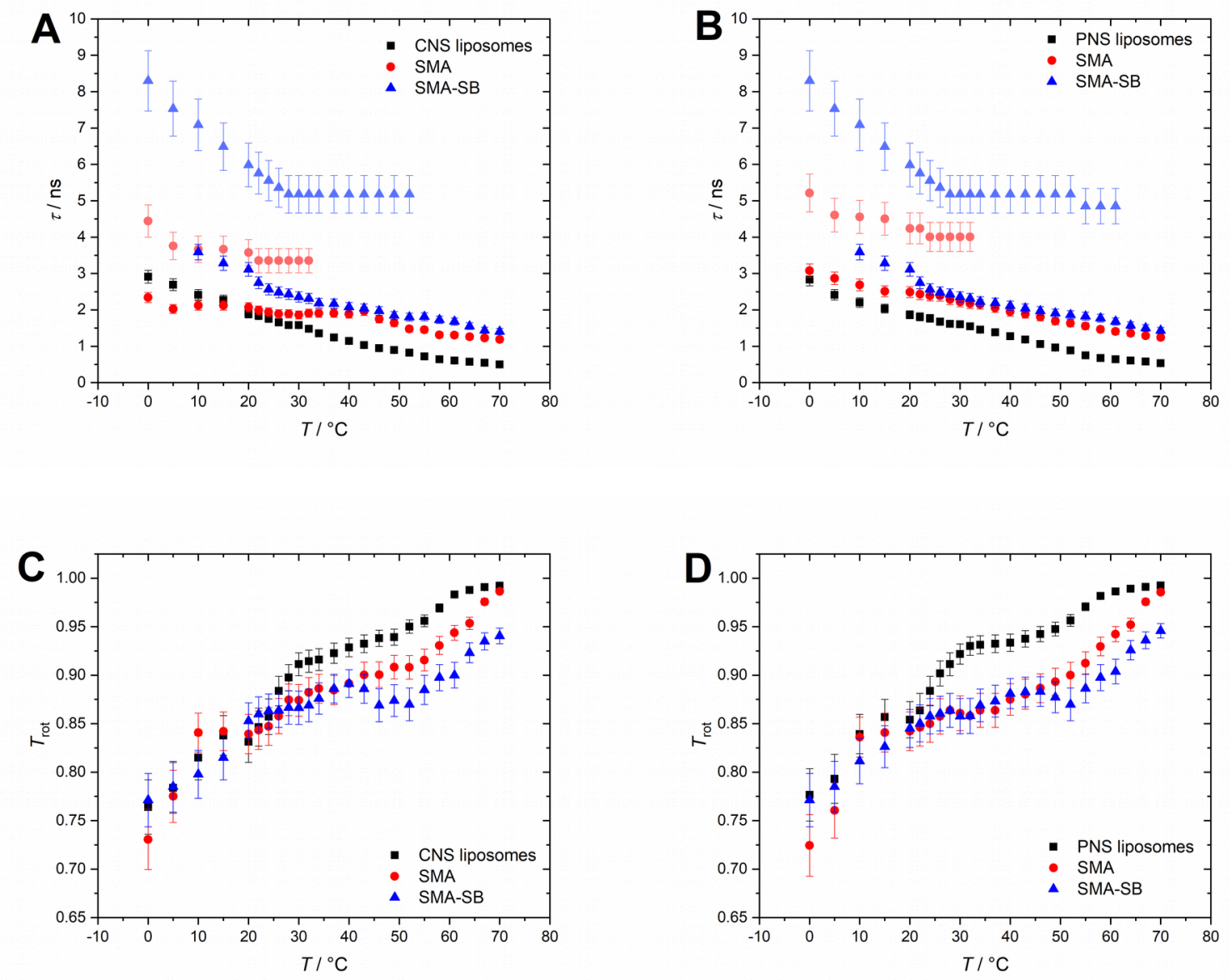

Figure 3. Simulation results of CW EPR spectra of 16DSPC (A/B) and 5DSPC (C/D) in liposomes and nanodiscs of the cytosolic CNS (A/C) and PNS (B/D) myelin composition. Shown are the rotational correlation time $\tau_{\mathrm{c}}(\mathbf{A} /$ B) of 16DSPC and the rotational anisotropy $T_{\text {rot }}(\mathbf{C} / \mathbf{D})$ of 5DSPC. The spectral component of slow rotation appearing in X-band CW EPR spectra of 16DSPC of SMALPs and SMA-SBLPs is presented in brightened colors to set it apart from the fast-rotating component.

Suitability of myelin-like nanodiscs as model system for protein studies

Successful preparation of nanodiscs with a (near) native lipid composition is only the first step necessary for in vitro myelin research. Generally, the suitability of the prepared systems for mimicking cytosolic myelin requires further investigations. To this end, we added myelin basic protein (MBP) to the myelin-like nanodiscs to test for MBP-membrane interaction and induced self-assembly. MBP is abundant in both CNS and PNS cytosolic myelin, and we have ample experience in the characterization of MBP in different charge variants in LUVs. ${ }^{12,52}$ As a suitable model protein, here we used bovine $\mathrm{MBP}^{26}$ which presents a similar amino acid sequence as human MBP. ${ }^{53}$

In the myelin sheath, MBP is incorporated into the multilayer system between both cytosolic leaflets of opposed bilayers. ${ }^{11-12}$ Since MBP acts as "molecular glue", holding together two lipid bilayers, ${ }^{11-12}$ it induces association of multiple lipid particles.
Therefore, its interactions with lipid model systems can be observed by light scattering techniques. To ensure comparability of all measurements, we added the same amount of bMBP to each sample resulting in equal protein concentration but differing lipid concentration and, thus, different lipid/protein ratio.

First, DLS of the nanodiscs with and without addition of bMBP was measured. The resulting size distributions for SMALPs of both PNS and CNS myelin-like nanodiscs are shown in Figure 4 (for both other polymers and comparison with pure bMBP see Figure S30). In the case of SMALPs, addition of bMBP has a strong effect on the size of scattering particles. The small nanodiscs disappear while new particles of hydrodynamic diameters of more than $1 \mu \mathrm{m}$ appear. While the vanishing of the small particles probably was induced by larger particles, which scatter much more strongly, the large particles additionally observed must be the result of aggregated nanodiscs. 
The bMBP alone exhibited a small fraction of aggregated protein. Those large particles, however, did not scatter to a similar extent as the mixtures of SMALPs and bMBP. Furthermore, mixtures of $\mathrm{bMBP}$ and the polymer without lipids do not contain particles of similar size (see Figure S30). Therefore, the observed interactions cannot originate from unspecific coulombic interactions between anionic polymer

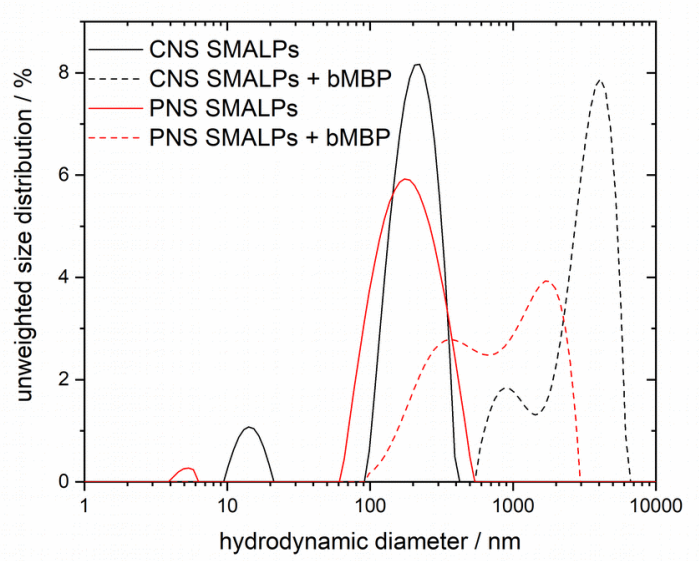

Figure 4. Size distribution of SMALPs of the cytosolic CNS and PNS myelin lipid composition with and without bMBP as observed with DLS measurements.

and cationic bMBP alone. For SMA-SBLPs, by contrast, no observable size change of the lipid particles is induced by the protein (see Figure S30).

From DLS measurements, it can be concluded, that only in myelin-like SMALPS bMBP can execute its native function of stacking lipid bilayers to a noticeable extent.

By measuring CW EPR spectra of myelin-like nanodiscs containing 16DSPC or 5DSPC with bMBP it is possible to study the protein's effect on the bound lipids. The spectra of all nanodisc samples are shown in Figure S31 and Figure $\mathbf{S 3 2}$ in the Supporting Information.

We found that addition of the protein induced no changes in lipid mobility and hydration for all studied systems. One would typically assume in CW EPR studies of interactions between penetrating proteins and lipids that an additional spectral component after addition of the protein becomes observable. ${ }^{54}$ This is not the case here after addition of bMBP. However, bMBP had an effect on the spectral intensity in some cases. When the protein is added to myelin-like nanodiscs the double integral value has to be reduced to $50 \%$ of the pure systems due to dilution. In both SMALP systems and SMA-SBLPs containing PNS lipids the double integral was reduced even further to $30-40 \%$ of the nanodiscs without protein while for DIBMALPs and CNS SMASBLPs it remained approximately at $50 \%$.

A decrease in spectral intensity can be induced by increased spin exchange interaction between the spin probes. ${ }^{55}$ If this interaction becomes strong enough, i.e., the spin probes collide directly, the spectral peaks can become broad enough to be indiscernible from the baseline. This effect could be induced by guest molecules such as proteins which preferably bind lipids or reject them if the model system is spatially confined.

In case of myelin-like SMALPs, the size of the nanodiscs limited the space available for the lipids to approximately $10 \mathrm{~nm}$. bMBP as a positively charged protein is known to preferably bind to negatively charged lipid surfaces. $^{26,}{ }^{56}$ Subsequently, it probably gathers negatively charged lipids in inhomogeneous membranes like the studied myelin-like composition. The used spin label lipids bear zwitterionic PC headgroups. Thus, it is possible that bMBP repelled or ignored the PC spin labels to some extent which is in accordance with literature showing weak interaction between bMBP and PC lipids. ${ }^{26,56}$ If the protein did not bind the spin probe lipids, they accumulate at the rim of the nanodisc and, hence, likely interact more frequently among themselves, which causes a broadening of the peaks.

While the addition of bMBP to myelin-like nanodiscs did not directly affect the mobility and hydration of the spin-labeled lipids, they could be pushed to the rim of the nanodisc and exhibit strong peak broadening which, thus, have decreased intensity. This indication of protein binding to the lipids was only found for myelin-like SMALPS and SMA-SBLPS containing PNS lipids. This may be due to a slightly higher binding affinity of bMBP to the more anionic PNS lipid mixture.

In conclusion, we have shown that myelinlike nanodiscs encapsulated by SMA are the only model systems that are able to bind bMBP in a native way. SMA-SBLPs only bind the protein if the lipid composition resembles the cytosolic PNS, which may be the result of changed lipid properties as discussed before. However, in PNS SMASBLPs bMBP was not able to stack the nanodiscs. The resulting model is depicted in .

\section{Conclusions}


In this study, we present the preparation of myelin-like nanodiscs with SMA containing either PNS or CNS cytosolic lipids. The preparation process involves solubilization experiments for each lipid mixture using DLS for nanodisc detection, separation of remaining liposomes with size exclusion chromatography followed by detection of resulting nanodiscs with DLS, again.

The myelin-like nanodiscs were studied regarding their lipid composition, the properties of the myelin lipids and their suitability to be used as model system for natural myelin in lab scale.
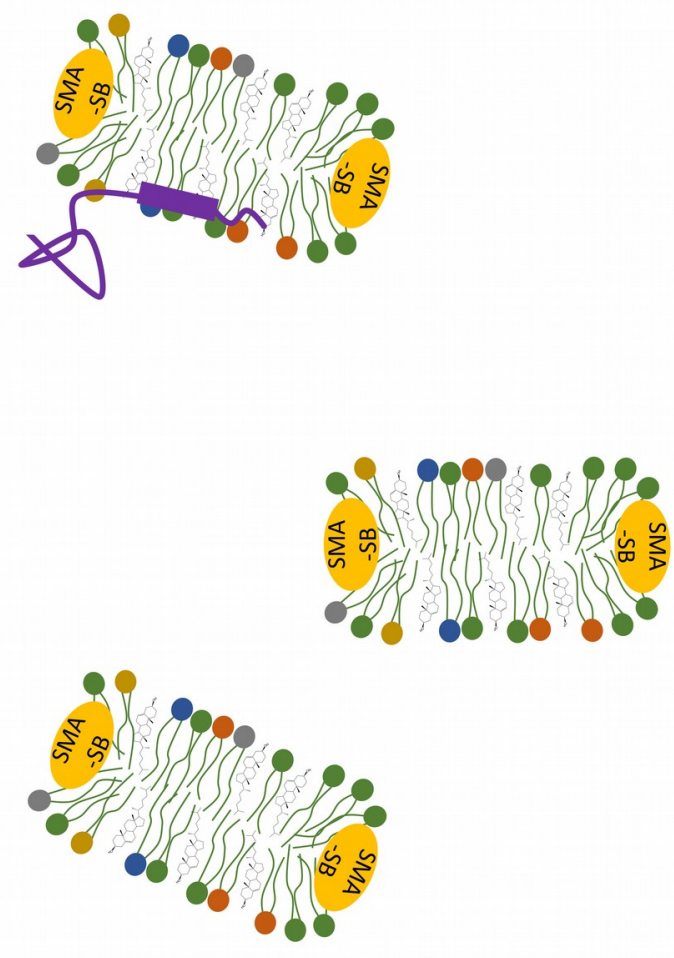

Our results suggest that the preparation in acceptable amount is possible with the polymers SMA and SMA-SB. Both polymers do not show any preference for distinct lipid headgroups or chains. However, only myelin-like SMALPs present lipid-protein interactions with the model protein bMBP while maintaining the protein's natural function.

By including the spin-labeled lipids 16DSPC and 5DSPC in our solubilization, we were able to characterize the effects of both SMA and SMA-SB on the lipids within the myelinlike nanodiscs either in the

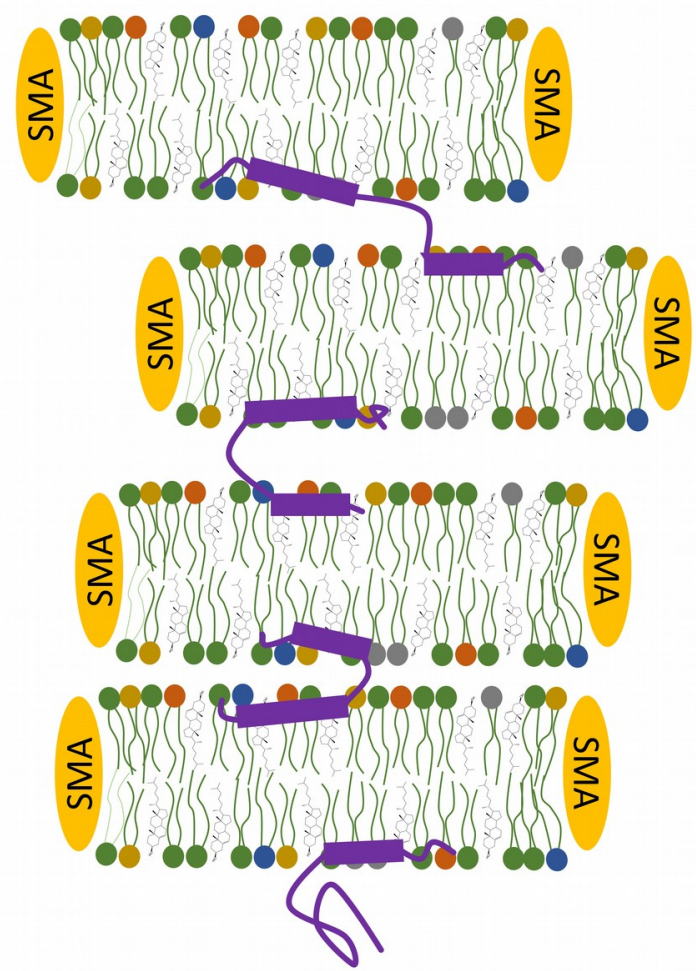

Figure 5. Suitability of SMALPS and SMA-SBLPs as model system for human myelin. While SMALPs of both cytosolic CNS and PNS lipid composition can bind bMBP and aggregate, only the PNS-like SMA-SBLPs can bind bMBP but, however, do not show aggregation. Note the different constraint both polymers exert onto the lipids as observed with CW EPR measurements. The myelin-like lipid composition is indicated by differently colored phospholipids as well as cholesterol. The sizes in this scheme are not to scale and size differences are exaggerated.

hydrophobic center of the bilayer or near the carbonyl groups. We find that both polymers exert steric constraints onto the hydrophobic part of the lipids while a small loosening effect is observable for the carbonyl-near membrane region. Both effects were significantly more pronounced in SMA-SBLPS and may have prevented the membrane stacking by bMBP. The third amphiphilic polymer in this study, DIBMA, was not able to solubilize myelin-like liposomes in a usable extent.
In future studies, the preparation process described herein will be further optimized and different proteins will be studied in combination with the myelin-like lipid composition. Therefore, even SMA-SB and DIBMA could be suitable for solubilization with different experimental parameters. The composition of the nanodiscs could be optimized using both cytosolic and extracellular lipids. If it is possible to prepare nanodiscs containing both the extracellular and the cytosolic leaflet this would enable even more nature-like research such as mimicking myelin with all 
major lipids and proteins in combination. When considering that the lipid composition of PNS and CNS myelin is challenging to reconstruct in nanodiscs, our work shows that more complex lipid model membrane systems are in general accessible through nanodiscs. We can furthermore study protein-lipid interactions in myelin and factors driving myelin formation or degradation using combinations of myelin proteins in a highly controlled lipid environment resembling myelin's cytoplasmic leaflet.

\section{ASSOCIATED CONTENT}

\section{Supporting Information.}

The Supporting Information is available free of charge at http://pubs.acs.org.

Details on CW EPR simulation and lipids, optimal polymer/lipid ratios, further DLS data, chromatograms, TEM micrographs, HPLC calibration, MS data, CW EPR data including spectra and simulation results, and DSC data

\section{AUTHOR INFORMATION}

\section{Corresponding Author}

* E-mail address: dariush.hinderberger@chemie.unihalle.de

Telephone number: +49 345 55-25230

Fax number: +49 345 55-27576

\section{Present Addresses}

David Haselberger: Institute of Physics, Martin Luther University (MLU) Halle-Wittenberg, BettyHeimann-Str. 7, 06120 Halle (Saale), Germany.

\section{Author Contributions}

M.H., T.H., L.M., M.D., C.V., S.K., P.L.K., C.S., and D.Hi. conceived and planned research; M.H., D.Ha., and M.D. contributed to sample preparation; M.H., D.Ha., T.H., and L.M. performed experiments; M.H., D.Ha., T.H., L.M., K.J., A.M., and D.Hi. analyzed data; M.H. carried out spectral simulations; M.H., T.H., L.M., K.J., S.K., P.L.K., C.S., and D.Hi. wrote the publication.

\section{Funding Sources}

M.H. thanks the Fonds der Chemischen Industrie $(\mathrm{FCl})$ for a Kekulé scholarship. T.H. and C.S. acknowledge funding from the Federal Ministry for Education and Research (BMBF, 03Z22HN22) and the European Regional Development Funds (EFRE, ZS/2016/04/78115). P.L.K. was supported by the Federal Ministry for Education and Research (BMBF, ZIK program) (Grant nos. 03Z22HN23 and 03COV04), the European Regional Development Funds for Saxony-Anhalt (grant no. EFRE: ZS/2016/04/78115), funding by Deutsche Forschungsgemeinschaft (DFG) (project number 391498659, RTG 2467) and the Martin-Luther University of Halle-Wittenberg. A.M. and P.L.K. were supported by International Graduate School AGRIPOLY, funded by the European Regional Development Fund (ERDF) and the Federal State Saxony-Anhalt with a scholarship to K.J.

\section{ACKNOWLEDGMENT}

We thank Annekatrin Rother, Gerd Hause, and Farzad Hamdi for their support in conducting electron microscopy (EM) and Fotis Kyrilis for his support in EM and SEC experiments.

\section{ABBREVIATIONS}

5DSPC, 1-palmitoyl-2-stearoyl-(5-doxyl)-sn-glycero3-phosphocholine, 16DSPC, 1-palmitoyl-2-stearoyl(16-doxyl)-sn-glycero-3-phosphocholine, bMBP, bovine myelin basic protein, CNS, central nervous system, CV, column volume, CW EPR, continuous wave electron paramagnetic resonance, $\mathrm{DI}$, double integral, DIBMA, diisobutylene/maleic acid copolymer, DLS, dynamic light scattering, DSC, differential scanning calorimetry, HPLC, high performance liquid chromatography, LPs, lipid particles (used in combination with the three polymers), MBP, myelin basic protein, MS, mass spectrometry, NMR, nuclear magnetic resonance, PC, phosphatidylcholine lipids, PE, phosphatidylethanolamine lipids, PI, phosphatidylinositol lipids, PS, phosphatidylserine lipids, PNS, peripheral nervous system, SEC, size exclusion chromatography, SM, sphingomyelin lipids, SMA, styrene/maleic acid copolymer, SMA-SB, styrene/maleic amide sulfobetaine copolymer, TEM, transmission electron microscopy, TLC, thin layer chromatography.

\section{REFERENCES}

1. Lassmann, H., Mechanisms of white matter damage in multiple sclerosis. Glia 2014, 62 (11), 18161830.

2. Nave, K. A.; Werner, H. B., Myelination of the nervous system: mechanisms and functions. Annu. Rev. Cell. Dev. Biol. 2014, 30, 503-533.

3. Niemann, A.; Berger, P.; Suter, U., Pathomechanisms of mutant proteins in Charcot-MarieTooth disease. Neuromolecular Med. 2006, 8 (1-2), 217-241.

4. Fraser, P. E.; Deber, C. M., Surface accessibility of 13C-labeled lysine residues in membrane-bound myelin basic protein. J. Biol. Chem. 1984, 259 (14), 8689-8692.

5. Sedzik, J.; Blaurock, A. E.; Höchli, M., Lipid/Myelin Basic Protein Multilayers A Model for the Cytoplasmic Space in Central Nervous System Myelin. J. Mol. Biol. 1984, 174, 385-409.

6. Shanshiashvili, L. V.; Suknidze, N. C.; Machaidze, G. G.; Mikeladze, D. G.; Ramsden, J. J., Adhesion and clustering of charge isomers of myelin basic protein at model myelin membranes. Arch. Biochem. Biophys. 2003, 419 (2), 170-177.

7. Walker, A. G.; Rumsby, M. G., The induction of liposome aggregation by myelin basic protein. Neurchem. Int. 1985, 7, 441-447.

8. Yurlova, L.; Kahya, N.; Aggarwal, S.; Kaiser, H. J.; Chiantia, S.; Bakhti, M.; Pewzner-Jung, Y.; Ben-David, O.; Futerman, A. H.; Brügger, B.; Simons, M., Selfsegregation of myelin membrane lipids in model membranes. Biophys. J. 2011, 101 (11), 2713-2720.

9. Hartline, D. K., What is myelin? Neuron Glia Biol. 2008, 4 (2), 153-163.

10. Boggs, J. M.; Moscarello, M. A., Structural organization of the human myelin membrane. Biochim. Biophys. Acta 1978, 515, 1-21. 
11. Bakhti, M.; Aggarwal, S.; Simons, M., Myelin architecture: zippering membranes tightly together. Cell. Mol. Life Sci. 2014, 71 (7), 1265-1277.

12. Tzakos, A. G.; Kursula, P.; Theodorou, V.; Troganis, A.; Tselios, T.; Svarnas, C.; Matsoukas, J.; Apostolopoulos, V.; Gerothanassis, I. P., Structure and function of the myelin proteins: current status and perspectives in relation to multiple sclerosis. Curr. Med. Chem. 2005, 12, 1569-1587.

13. Raasakka, A.; Ruskamo, S.; Kowal, J.; Han, H.; Baumann, A.; Myllykoski, M.; Fasano, A.; Rossano, R.; Riccio, P.; Bürck, J.; Ulrich, A. S.; Stahlberg, H.; Kursula, P., Molecular structure and function of myelin protein PO in membrane stacking. Sci. Rep. 2019, 9 (1), 642.

14. Knowles, T. J.; Finka, R.; Smith, C.; Lin, Y. P.; Dafforn, T.; Overduin, M., Membrane Proteins Solubilized Intact in Lipid Containing Nanoparticles Bounded by Styrene Maleic Acid Copolymer. J. Am. Chem. Soc. 2009, 131, 7484-7485.

15. Scheidelaar, S.; Koorengevel, M. C.; Pardo, J. D.; Meeldijk, J. D.; Breukink, E.; Killian, J. A., Molecular model for the solubilization of membranes into nanodisks by styrene maleic Acid copolymers. Biophys. J. 2015, 108 (2), 279-290.

16. Oluwole, A. O.; Danielczak, B.; Meister, A.; Babalola, J. O.; Vargas, C.; Keller, S., Solubilization of Membrane Proteins into Functional Lipid-Bilayer Nanodiscs Using a Diisobutylene/Maleic Acid Copolymer. Angew. Chem. Int. Ed. 2017, 56 (7), 19191924.

17. Ravula, T.; Ramadugu, S. K.; Di Mauro, G.; Ramamoorthy, A., Bioinspired, Size-Tunable SelfAssembly of Polymer-Lipid Bilayer Nanodiscs. Angew. Chem. Int. Ed. 2017, 56 (38), 11466-11470.

18. Yasuhara, K.; Arakida, J.; Ravula, T.; Ramadugu, S. K.; Sahoo, B.; Kikuchi, J. I.; Ramamoorthy, A., Spontaneous Lipid Nanodisc Fomation by Amphiphilic Polymethacrylate Copolymers. J. Am. Chem. Soc. 2017, 139 (51), 18657-18663.

19. Grethen, A.; Oluwole, A. O.; Danielczak, B.; Vargas, C.; Keller, S., Thermodynamics of nanodisc formation mediated by styrene/maleic acid (2:1) copolymer. Sci. Rep. 2017, 7 (1), 11517.

20. Eisermann, J.; Hoffmann, M.; Schöffmann, F. A.; Das, M.; Vargas, C.; Keller, S.; Hinderberger, D., Molecular-Level Interactions of Nanodisc-Forming Copolymers Dissected by EPR Spectroscopy. Macromol. Chem. Phys. 2021, accepted manuscript.

21. Hoffmann, M.; Eisermann, J.; Schöffmann, F. A.; Das, M.; Vargas, C.; Keller, S.; Hinderberger, D., Influence of Different Polymer Belts on Lipid Properties in Nanodiscs Characterized by CW EPR Spectroscopy. Biochim. Biophys. Acta Biomembranes 2021, submitted manuscript.

22. Dörr, J. M.; Scheidelaar, S.; Koorengevel, M. C.; Dominguez, J. J.; Schäfer, M.; van Walree, C. A.; Killian, J. A., The styrene-maleic acid copolymer: a versatile tool in membrane research. Eur. Biophys. J. 2016, 45 (1), 3-21.

23. Oluwole, A. O.; Klingler, J.; Danielczak, B.; Babalola, J. O.; Vargas, C.; Pabst, G.; Keller, S., Formation of Lipid-Bilayer Nanodiscs by Diisobutylene/Maleic Acid (DIBMA) Copolymer. Langmuir 2017, 33 (50), 14378-14388.

24. Smith, A. A. A.; Autzen, H. E.; Faust, B.; Mann, J. L.; Muir, B. W.; Howard, S.; Postma, A.; Spakowitz, A. J.; Cheng, Y.; Appel, E. A., Lipid Nanodiscs via Ordered Copolymers. Chem 2020, 6 (10), 2782-2795.

25. Inouye, H.; Kirschner, D. A., Membrane interactions in nerve myelin: determination of surface charge from biochemical data. Biophys. J. 1988, 53 247-260.

26. Widder, K.; Träger, J.; Kerth, A.; Harauz, G.; Hinderberger, D., Interaction of Myelin Basic Protein with Myelin-like Lipid Monolayers at Air-Water Interface. Langmuir 2018, 34 (21), 6095-6108.

27. Bligh, E. G.; Dyer, W. J., A rapid method of total lipid extraction and purification. Can. J. Biochem. Physiol. 1959, 37, 911-917.

28. Becart, J.; Chevalier, C.; Biesse, J. P., Quantitative analysis of phospholipids by HPLC with a light scattering evaporating detector - application to raw materials for cosmetic use. J. High Resolut. Chromatogr. 1990, 13, 126-129.

29. Hofmann, T.; Barth, M.; Seiffert, S.; Meister, A.; Schmidt, C., in preparation $\mathbf{2 0 2 1}$.

30. Stoll, S.; Schweiger, A., EasySpin, a comprehensive software package for spectral simulation and analysis in EPR. J. Magn. Reson. 2006, 178 (1), 42-55

31. Colbasevici, A.; Voskoboynikova, N.; Orekhov, P. S.; Bozdaganyan, M. E.; Karlova, M. G.; Sokolova, O. S.; Klare, J. P.; Mulkidjanian, A. Y.; Shaitan, K. V.; Steinhoff, H. J., Lipid dynamics in nanoparticles formed by maleic acid-containing copolymers: EPR spectroscopy and molecular dynamics simulations. Biochim. Biophys. Acta Biomembr. 2020, 1862 (5), 183207.

32. Marsh, D., Distinct Populations in Spin-Label EPR Spectra from Nitroxides. J. Phys. Chem. B 2018, 122 (23), 6129-6133.

33. Ayee, M. A.; Levitan, I., Paradoxical impact of cholesterol on lipid packing and cell stiffness. Front. Biosci. 2016, 21, 1245-1259.

34. Veatch, S. L.; Keller, S. L., Miscibility phase diagrams of giant vesicles containing sphingomyelin. Phys. Rev. Lett. 2005, 94 (14), 148101.

35. Danielczak, B.; Keller, S., Collisional lipid exchange among DIBMA-encapsulated nanodiscs (DIBMALPs). Eur. Polym. J. 2018, 109, 206-213.

36. Danielczak, B.; Keller, S., Lipid exchange among polymer-encapsulated nanodiscs by timeresolved Förster resonance energy transfer. Methods 2020, 180, 27-34.

37. Hazell, G.; Arnold, T.; Barker, R. D.; Clifton, L. A.; Steinke, N. J.; Tognoloni, C.; Edler, K. J., Evidence of Lipid Exchange in Styrene Maleic Acid Lipid Particle (SMALP) Nanodisc Systems. Langmuir 2016, 32 (45), 11845-11853.

38. Vargas, C.; Arenas, R. C.; Frotscher, E.; Keller, S., Nanoparticle self-assembly in mixtures of phospholipids with styrene/maleic acid copolymers or fluorinated surfactants. Nanoscale 2015, 7 (48), 2068520696.

39. Cuevas Arenas, R.; Klingler, J.; Vargas, C.; Keller, S., Influence of lipid bilayer properties on nanodisc formation mediated by styrene/maleic acid copolymers. Nanoscale 2016, 8 (32), 15016-26.

40. Medina-Carmona, E.; Varela, L.; Hendry, A. C.; Thompson, G. S.; White, L. J.; Boles, J. E.; Hiscock, J. R.; Ortega-Roldan, J. L., A quantitative assay to study the lipid selectivity of membrane-associated systems using solution NMR. Chem. Commun. 2020, 56 (78), 1166511668.

41. Danielczak, B.; Meister, A.; Keller, S., Influence of $\mathrm{Mg}(2+)$ and $\mathrm{Ca}(2+)$ on nanodisc formation by diisobutylene/maleic acid (DIBMA) copolymer. Chem. Phys. Lipids 2019, 221, 30-38.

42. Scheidelaar, S.; Koorengevel, M. C.; van Walree, C. A.; Dominguez, J. J.; Dörr, J. M.; Killian, J. A., Effect of Polymer Composition and $\mathrm{pH}$ on Membrane 
Solubilization by Styrene-Maleic Acid Copolymers. Biophys. J. 2016, 111 (9), 1974-1986.

43. Gerlock, J. L., Determination of Free Radicals in Polymer Films by Electron Spin Resonance Spectrometry. Anal. Chem. 1983, 55, 1520-1522.

44. Bauer, D. R.; Gerlock, J. L., ESR Studies of Photooxidation and Stabilization of Polymer Coatings. In Advanced ESR Methods in Polymer Research, Schlick, S., Ed. John Wiley \& Sons, Inc.: Hoboken, 2006; pp 255278.

45. Kurad, D.; Jeschke, G.; Marsh, D., Lipid Membrane Polarity Profiles by High-Field EPR. Biophys. J. 2003, 85, 1025-1033.

46. Vartorelli, M. R.; Garay, A. S.; Rodrigues, D. E., Spin-labeled Stearic Acid Behavior and Perturbations on the Structure of a Gel-Phase-Lipid Bilayer in Water: 5-, 12- and 16-SASL. J. Phys. Chem. B 2008, 112, 1683016842.

47. Stepien, P.; Polit, A.; Wisniewska-Becker, A., Comparative EPR studies on lipid bilayer properties in nanodiscs and liposomes. Biochim. Biophys. Acta 2015, 1848 (1 Pt A), 60-66.

48. Erilov, D. A.; Bartucci, R.; Guzzi, R.; Shubin, A. A.; Maryasov, A. G.; Marsh, D.; Dzuba, S. A.; Sportelli, L., Water Concentration Profiles in Membranes Measured by ESEEM of Spin-Labeled Lipids. J. Phys. Chem. B 2005, 109, 12003-12013.

49. Eisermann, J.; Prager, L.; Hinderberger, D., Solvent and concentration effects on highly defined, colloid-like ionic clusters in solution. Phys. Chem. Chem. Phys. 2018, 20 (3), 1421-1430.

50. Jamshad, M.; Grimard, V.; Idini, I.; Knowles, T. J.; Dowle, M. R.; Schofield, N.; Sridhar, P.; Lin, Y. P.; Finka, R.; Wheatley, M.; Thomas, O. R.; Palmer, R. E.;
Overduin, M.; Govaerts, C.; Ruysschaert, J. M.; Edler, K. J.; Dafforn, T. R., Structural analysis of a nanoparticle containing a lipid bilayer used for detergent-free extraction of membrane proteins. Nano Res. 2015, 8 (3), 774-789.

51. Kleinschmidt, J. H.; Mahaney, J. E.; Thomas, D. D.; Marsh, D., Interaction of Bee Venom Melittin with Zwitterionic and Negatively Charged Phospholipid Bilayers: A Spin-Label Electron Spin Resonance Study. Biophys. J. 1997, 72, 767-778.

52. Kattnig, D. R.; Bund, T.; Boggs, J. M.; Harauz, G.; Hinderberger, D., Lateral self-assembly of $18.5-\mathrm{kDa}$ myelin basic protein (MBP) charge component-C1 on membranes. Biochim. Biophys. Acta 2012, 1818 (11), 2636-2647.

53. Harauz, G.; Musse, A. A., A tale of two citrullines - structural and functional aspects of myelin basic protein deimination in health and disease. Neurochem. Res. 2007, 32 (2), 137-158.

54. Marsh, D., Electron spin resonance in membrane research: protein-lipid interactions. Methods 2008, 46 (2), 83-96.

55. Schneider, D. J.; Freed, J. H., Calculating Slow Motional Magnetic Resonance Spectra. In Biological Magnetic Resonance, Berliner, L. J.; Reuben, J., Eds. Plenum Press: New York, 1989; Vol. 8, Spin Labeling Theory and Applications, pp 1-76.

56. Polverini, E.; Arisi, S.; Cavatorta, P.; Berzina, T.; Cristofolini, L.; Fasano, A.; Riccio, P.; Fontana, M. P., Interaction of Myelin Basic Protein with Phospholipid Monolayers: Mechanism of Protein Penetration. Langmuir 2003, 19, 872-877.

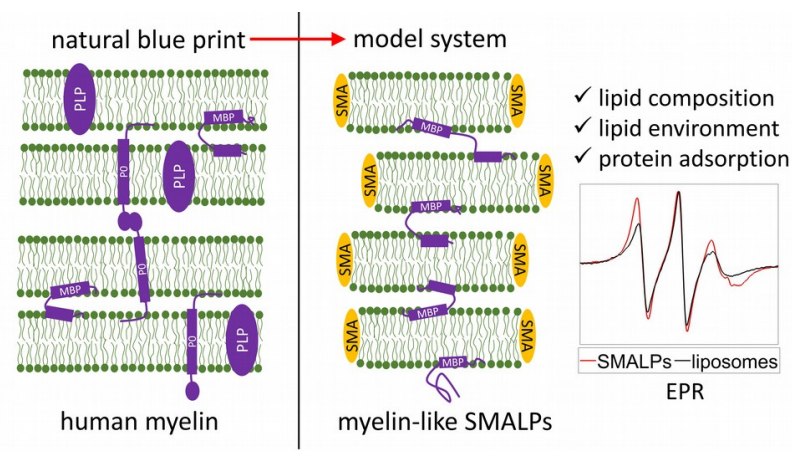

For Table of Contents only. 